\title{
Using Galerkin Method for Solving Linear Optimal Control Problems
}

\begin{abstract}
I. A. Pankratov
Saratov State University, 83, Astrakhanskaya str., Saratov, 410012, Russia, PankratovlA@info.sgu.ru

The linear optimal control problem is considered. Duration of the controlled process is fixed. It is necessary to minimize the functional, that characterizes the energy consumption. A method of constructing an approximate solution based on the Galerkin method is proposed. Examples of numerical solutions of the problem are given.
\end{abstract}

Key words: optimal control, linear system, Galerkin method.

This work was supported by the Russian Foundation for Basic Research (project no. 12-01-00165-a).

\section{References}

1. Pontriagin L. S., Boltianskii V. G., Gamkrelidze R. V., Mishchenko E. F. Matematicheskaia teoriia optimal'nykh protsessov [The mathematical theory of optimal processes]. Moscow, Nauka, 1983, 393 p. (in Russian).

2. Roitenberg Ya. N. Avtomaticheskoe upravlenie [Automatic control]. Moscow, Nauka, 1983, 393 p. (in Russian).

3. Moiseev N. N. Chislennye metody v teorii optimal'nykh sistem [Numerical methods in the theory of optimal systems]. Moscow, Nauka, 1971, 424 p. (in Russian).

4. Fedorenko R. P. Priblizhennoe reshenie zadach optimal'nogo upravleniia [Approximate solution of optimal control problems]. Moscow, Nauka, 1978, 488 p. (in Russian).

5. Vasil'ev F. P. Chislennye metody resheniia ekstremal'nykh zadach [Numerical methods for solving extremal problems]. Moscow, Nauka, 1988, 552 p. (in Russian).

6. Zienkiewicz O., Morgan K. Finite elements and ap-

proximation. New York, Chichester, Brisbane, Toronto, John Wiley and Sons, 1983, 328 p.

7. Pankratov I. A., Sapunkov Ya. G., Chelnokov Yu. N. About a problem of spacecraft's orbit optimal reorientation. Izv. Saratov Univ. (N.S.), Ser. Math. Mech. Inform., 2012, vol. 12, iss. 3, pp. 87-95 (in Russian).

8. Pankratov I. A., Sapunkov Ya. G., Chelnokov Yu. N. Solution of a problem of spacecraft's orbit optimal reorientation using quaternion equations of orbital system of coordinates orientation. Izv. Saratov Univ. (N. S.), Ser. Math. Mech. Inform., 2013, vol. 13, iss. 1, pt. 1, pp. 87-95 (in Russian).

9. Chelnokov Yu. N. The use of quaternions in the optimal control problems of motion of the center of mass of a spacecraft in a newtonian gravitational field: III. Cosmic Research, 2003, vol. 41, no. 5, pp. 460-477. DOI 10.1023/A:1026098216710.

УДК 532.517.2:534.2

\section{ДВУХСЛОЙНОЕ ТЕЧЕНИЕ РАСПЛАВОВ ПОЛИМЕРОВ В КАНАЛАХ ФИЛЬЕР}

\author{
Б. А. Снигерев ${ }^{1}$, Ф. Х. Тазюков ${ }^{2}$ \\ ${ }^{1}$ Доктор технических наук, ведущий научный сотрудник лаборатории моделирования технологических процессов, Институт \\ механики и машиностроения Казанского научного центра РАН, snigerev@ mail.knc.ru \\ ${ }^{2}$ Доктор технических наук, профрессор кафредры теоретической механики и сопротивления материалов, Казанский нацио- \\ нальный исследовательский технологический университет, tazyukov@ mail.ru
}

Работа посвящена численному моделированию двухслойного течения нелинейно-вязких жидкостей в каналах фиильер. Движение жидкостей описывается уравнениями сохранения массы и импульса, дополненными реологическим уравнением состояния нелинейно-вязкой жидкости по модели Карро. Приводится методика численного решения задачи на основе метода конечных элементов. Исследована картина распределения скоростей жидкости, давления, напряжений, положения границы раздела в двухслойном потоке в зависимости от реологических свойств жидкости и режимов течения.

Ключевые слова: нелинейно-вязкая жидкость, граница раздела, двухслойное течение.

\section{ВВЕДЕНИЕ}

Слоистые, или стратифицированные, течения жидкостей давно привлекают внимание исследователей. Очень актуальными являются исследования, связанные с проблемами транспортировки сырой нефти по трубопроводам. Известны способы снижения давления, необходимого для перекачки нефти по трубам, путем добавления к нефти полимерного раствора, который оттесняясь к стенкам трубы, 
формирует двухслойный поток. В работе [1] рассматривается кольцевое течение двух несмешивающихся жидкостей в трубопроводе, когда в ядре потока движется основная жидкость с произвольной кривой течения, а в пристенном слое маловязкая не ньютоновская жидкость. Получены аналитические зависимости для расходов обеих жидкостей от перепада давления для двухслойного течения, состоящего из основного течения в трубе, дополненного кольцевым течением маловязкой жидкости в пристенном слое. Экспериментальное изучение влияния пристенного слоя маловязкой жидкости на расход высоковязкой не ньютоновской жидкости в трубопроводе проводится в работе [2].

В ряде работ проведены теоретические исследования с целью анализа причин неустойчивости сдвигового течения двухслойных систем, состоящих из упругих вязких жидкостей, когда в качестве реологических уравнений состояния используются уравнения не ньютоновских жидкостей $[3,4]$. Неустойчивость слоистого течения выражается через колебания координаты поверхности раздела относительно оси потока. Показано, что нестабильная форма поверхности раздела характерна для течений, у которых существенно различны вязкости и нормальные напряжения компонентов потока на линии их контакта. Но наиболее активно стимулировали развитие стратифицированных течений проблемы, возникающие при производстве слоистых полимерных пленок и биокомпонентных волокон методом соэкструзии, когда два или более потока расплава продавливают через формующую головку совместно [3-6]. Надо заметить, что предложенные математические модели не касаются исследования течения на этапе формирования потока, то есть области течения на начальном участке канала, где происходит образование совместного течения двух разнородных жидкостей.

В настоящей работе исследуется установившееся несмешивающееся двухслойное течение не ньютоновских жидкостей, образующееся в результате слияния двух потоков в плоском канале с образованием границы раздела течения.

\section{1. ПОСТАНОВКА ЗАДАЧИ}

Рассматривается безволновое неизотермическое течение двух несмешивающихся жидкостей в сходящемся канале, схема которого показана на рис. 1.

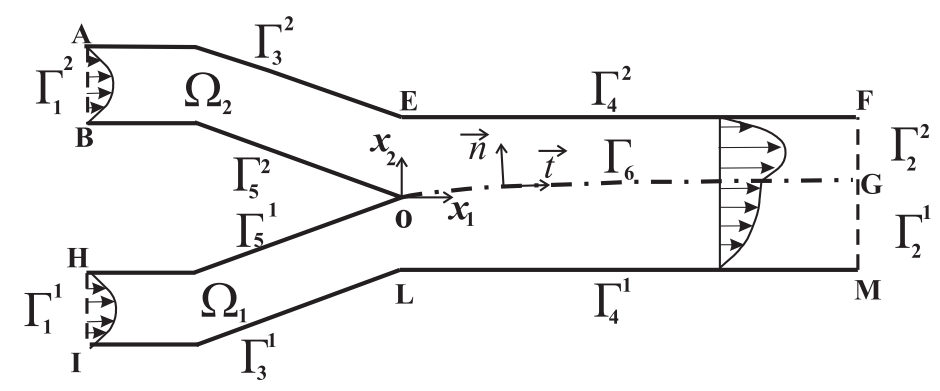

Рис. 1. Схема расчетной области двухслойного потока жидкости

Первая жидкость с объемным расходом $Q^{1}$ подается на входе $\Gamma_{1}^{1}(\mathrm{IH})$, вторая на входе $\Gamma_{1}^{2}(\mathrm{AB})$ с расходом $Q^{2}$, после схождения потоков в сечении EOL образуется совместное течение жидкостей с границей разделения потоков $\Gamma_{6}(\mathrm{OG})$. Область течения жидкости в нижнем слое IHOGM обозначается как $\Omega_{1}$, а в верхнем BAEFGO соответственно $\Omega_{2}$. Течение не ньютоновских жидкостей в каждой из областей $\Omega_{1,2}$ описывается уравнениями сохранения количества движения и неразрывности в виде

$$
\begin{gathered}
\varrho^{m}\left(\frac{\partial u_{i}^{m}}{\partial t}+u_{j}^{m} \frac{\partial u_{i}^{m}}{\partial x_{j}}\right)=\frac{\partial \sigma_{i j}^{m}}{\partial x_{j}}, \\
\frac{\partial u_{i}^{m}}{\partial x_{i}}=0, \quad i, j=1,2, \quad m=1,2 .
\end{gathered}
$$

Система уравнений (1)-(2) замыкается реологическим уравнением состояния нелинейно-вязкой жидкости в форме степенного закона [5]

$$
\begin{gathered}
\sigma_{i j}^{m}=-p^{m} \delta_{i j}+\tau_{i j}^{m}, \\
\tau_{i j}^{m}=\eta^{m}\left(I_{2}\right) D_{i j}, \quad D_{i j}=\frac{1}{2}\left(\frac{\partial u_{i}}{\partial x_{j}}+\frac{\partial u_{j}}{\partial x_{i}}\right), \\
\eta^{m}\left(I_{2}\right)=\mu_{0}\left(I_{2}\right)^{\frac{k-1}{2}} .
\end{gathered}
$$


В (1)-(3) $\varrho^{m}-$ плотность, $\sigma_{i j}^{m}-$ компоненты полного тензора напряжений, $p^{m}-$ давление, $x_{i}-$ декартовы координаты, $u_{i}^{m}-$ компоненты скорости для жидкости с индексом $m, \delta_{i j}-$ компоненты единичного тензора, $\tau_{i j}^{m}-$ компоненты тензора девиатора напряжений, $\eta^{m}\left(I_{2}\right)-$ эффективная вязкость жидкости, $I_{2}-$ второй инвариант тензора скоростей деформаций, $D_{i j}-$ компоненты тензора скоростей деформаций, $\mu_{0}-$ показатель консистенции жидкости (приведенная вязкость), $k-$ параметр модели.

В начальный момент времени граница раздела слоёв $\Gamma_{6}$ имеет плоскую горизонтальную форму. На входных участках $\Gamma_{1}^{m}$ задается профиль осевой скорости, характерный для установившегося течения не ньютоновской жидкости в плоском канале. На твердых стенках $\Gamma_{3,4,5}^{m}$ выполняются условия прилипания, а на выходных границах $\Gamma_{2}^{m}$ ставятся условия установившегося потока. На подвижной границе раздела слоев жидкости $\Gamma_{6}$ должно выполняться кинематическое условие. Кроме того, должны выполняться динамические условия, выражающиеся в равенстве касательных напряжений жидкости с каждой стороны границы раздела, а нормальные напряжения имеют разрыв [6].

Таким образом, граничные условия запишутся в виде

$$
\begin{aligned}
& \Gamma_{1}^{m}: \quad u_{1}^{m}=U_{0}^{m}\left(x_{2}\right), \quad u_{2}^{m}=0, \quad p^{m}=p_{0}^{m} ; \\
& \Gamma_{2}^{m}: \quad \partial u_{1}^{m} / \partial x_{1}=0 ; \\
& \Gamma_{3}^{m}, \Gamma_{4}^{m}, \quad \Gamma_{5}^{m}: u_{1}^{m}=u_{2}^{m}=0 ; \\
& \Gamma_{6}: \quad u_{i}^{m} \cdot n_{i}=0, \quad u_{i}^{1} \cdot t_{i}=u_{i}^{2} \cdot t_{i}, \\
& \sigma_{i j}^{1} \cdot t_{j}=\sigma_{i j}^{2} \cdot t_{j}, \quad \sigma_{i j}^{1} \cdot n_{j}-\sigma_{i j}^{2} \cdot n_{j}=2 \gamma H n_{j}
\end{aligned}
$$

Здесь $n_{i}, t_{j}$ - компоненты единичной нормали и касательной к поверхности $\Gamma_{6}$, условия на $\Gamma_{6}$ записаны в локальной декартовой системе координат, связанной с каждой точкой свободной поверхности, $\gamma$ - коэффициент поверхностного натяжения, $H-$ главная кривизна поверхности раздела.

В двумерном случае поверхность раздела описывается уравнением $F\left(x_{1}, x_{2}, t\right)=0$, тогда выполняется следующее условие [6]:

$$
\frac{\partial F}{\partial t}+u_{i}^{m} \frac{\partial F}{\partial x_{i}}=0
$$

\section{2. ЧИСЛЕННОЕ РЕШЕНИЕ ЗАДАЧИ}

Для решения уравнений (1)-(3) с граничными условиями (4) используется метод конечных элементов [7]. На каждом шаге по времени расчет проводится в два этапа, когда решаются исходные уравнения в областях $\Omega_{1}$ и $\Omega_{2}$ соответственно. Для получения матричных уравнений применяется стандартная процедура Галёркина [8]. В качестве конечных элементов используются четырехугольные элементы с квадратичной интерполяцией для переменных компонент скорости $u_{i}$, линейной для давления $p$, компонент девиатора тензора напряжений $\tau_{i j}$. Для аппроксимации деформируемых границ применяется конечно-элементная методика, базирующаяся на эйлеровом подходе и заключающаяся в локальной перестройке конечно-элементной сетки вблизи движущихся границ. Для реализации граничных условий на подвижной границе раздела $\Gamma_{6}^{n}$ на $n$ шаге по времени, определенной с помощью конечно-элементной аппроксимации, применяется линеаризованная итерационная процедура, когда граничные условия (4) заменяются для области расчета $\Omega_{1}^{n+1}$ на следующие:

$$
\begin{gathered}
\left.u_{i}^{1} \cdot t_{i}\right|^{n+1}=\left.u_{i}^{2} \cdot t_{i}\right|^{n},\left.\quad u_{i}^{1} \cdot n_{i}\right|^{n+1}=0, \\
\left.\sigma_{i j}^{1} \cdot n_{j}\right|^{n+1}=\left.\sigma_{i j}^{2} \cdot n_{j}\right|^{n}+2 \gamma H n_{j}, \\
\left.\sigma_{i j}^{1} \cdot t_{j}\right|^{n+1}=\left.\sigma_{i j}^{2} \cdot t_{j}\right|^{n} .
\end{gathered}
$$

Значения переменных на $n$ итерации в правой части записанных соотношений вычисляются на совместной границе по значениям из области $\Omega_{2}^{n}$. Затем решается аналогичная задача для области $\Omega_{2}^{n+1}$ с граничными условиями на $\Gamma_{6}^{n}$, в которых правые части соотношений (6) вычисляются по решению задачи (1)-(4) на $n$ итерации для области $\Omega_{1}^{n+1}$. После первого этапа определяется поле скоростей, давлений для обоих областей на $n+1$ итерации. Затем с помощью численной аппроксимации кинематического условия (5) определяется положение границы раздела $F^{n+1}$ на $n+1$ итерации. Далее, возможен переход к первому этапу с известным новым положением границы и решением задачи на предыдущей итерации для всей области течения. Итерации продолжаются до выполнения сходимости 
решения, которое заключается в определении установившегося положения границы, определяющегося как $\left|\frac{F^{n+1}-F^{n}}{F^{n}}\right|<=\varepsilon_{1}$, удовлетворении граничных условий для скорости и напряжений на подвижной границе раздела $\Gamma_{6}:\left|u_{i}^{1} \cdot t_{i}\right|^{n+1}-\left|u_{i}^{2} \cdot t_{i}\right|^{n}<=\varepsilon_{2},\left|\sigma_{i j}^{1} \cdot t_{j}\right|^{n+1}-\left|\sigma_{i j}^{1} \cdot t_{j}\right|^{n}<=\varepsilon_{2}$, где $\varepsilon_{1}=10^{-3}$, $\varepsilon_{2}=10^{-4}$.

\section{3. РЕЗУЛЬТАТЫ ЧИСЛЕННЫХ РАСЧЕТОВ}

Рассмотрим двухслойное течение не ньютоновских жидкостей в канале с шириной в области совместного течения $h=2 \cdot 10^{-3}$ м при расходах $Q^{1}=5 \cdot 10^{-9} \mathrm{~m}^{3} / \mathrm{c}, Q^{2}=20 \cdot 10^{-9} \mathrm{~m}^{3} / \mathrm{c}$. Значения физических констант для компонентов потока следующие : $\varrho^{1}=930$ кг $/$ м $^{3}, \mu_{0}^{1}=10010$ Па·с, $k^{1}=0.75$, $\varrho^{2}=980$ кг $/ \mathrm{м}^{3}, \mu_{0}^{2}=10020$ Па·с, $k^{2}=0.75$.

В случае двухслойного течения положение границы раздела определяется величинами расходов и их отношением, а также отношением величин вязкостей расплавов потоков [2]. Обозначим $l_{1}, l_{2}$ как ширина потоков для нижнего и верхнего слоя соответственно $\left(l_{1}+l_{2}=h\right)$. Введем безразмерную величину $h_{f}=l_{2} /(h / 2)$, показывающую степень изменения ширины потока при совместном течении в канале. Влияние расходов на положение границы раздела сред показано на рис. 2, где приведено распределение $h_{f}$ для отношений расходов $Q^{1} / Q^{2}: 1-Q^{1} / Q^{2}=5,2-Q^{1} / Q^{2}=10,3-Q^{1} / Q^{2}=20$. Значения $h_{f}$ для установившегося потока составляют $h_{f}=1.29,1.73,1.96$. Для определения влияния различия вязкости на положение границы раздела рассчитывались течения в условиях постоянства расходов в нижнем и верхнем слое $Q^{1}=Q^{2}$. Влияние вязкости определялось в условиях, когда вязкость в нижнем слое остается равной $\mu_{0}^{1}=10010$ Па.с, $k^{1}=0.75$, а в верхнем слое коэффициент $\mu_{0}^{1}$ увеличивается, при этом отношение $\mu_{0}^{2} / \mu_{0}^{1}$ равно $\mu_{0}^{2} / \mu_{0}^{1}=5,10,20$. Поскольку вязкость нижнего слоя жидкости в области совместного течения выше, чем в верхнем, то это приводит к смещению положения границы раздела от оси канала к верхней стенке. Дальнейшее увеличение $\mu_{0}^{2} / \mu_{0}^{1}$ приводит к росту отклонения, что иллюстрирует рис. 3. На рис. 3 показано распределение $h_{f}$ для трех вязкостных режимов: $1-\mu_{0}^{2} / \mu_{0}^{1}=5,2-\mu_{0}^{2} / \mu_{0}^{1}=10,3-\mu_{0}^{2} / \mu_{0}^{1}=20$.

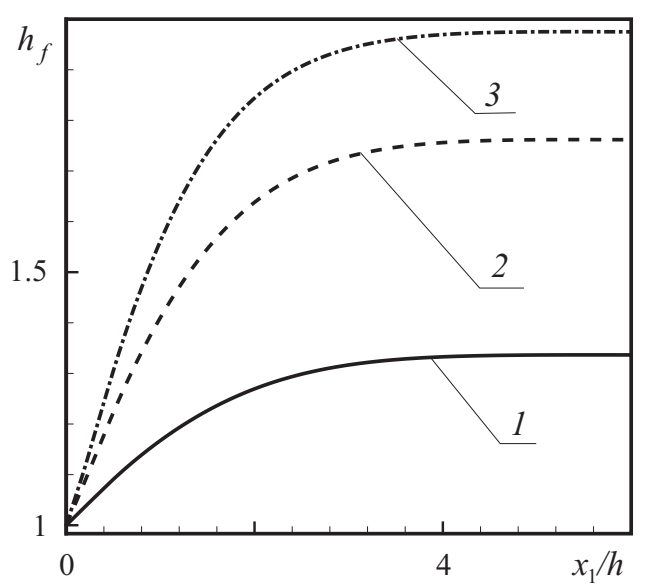

Рис. 2. Профили границы раздела жидкостей при различных $Q^{1} / Q^{2}: 1-Q^{1} / Q^{2}=5,2-$ $Q^{1} / Q^{2}=10,3-Q^{1} / Q^{2}=20$

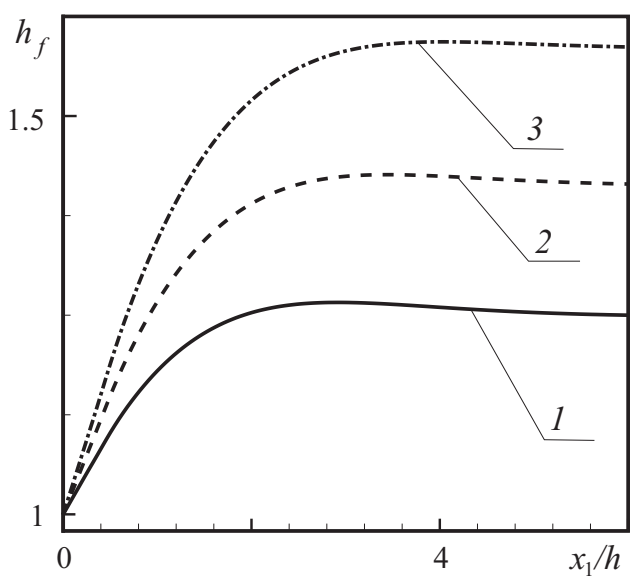

Рис. 3. Профили границы раздела жидкостей при различных $\mu_{0}^{1} / \mu_{0}^{2}: 1-\mu_{0}^{2} / \mu_{0}^{1}=5,2-$ $\mu_{0}^{2} / \mu_{0}^{1}=10,3-\mu_{0}^{2} / \mu_{0}^{1}=20$

На рис. 4 показаны установившиеся профили скорости $u_{1} / U_{0}$ двухслойного течения в сечении $x_{1} / h=3.0$ при отношении расходов $Q^{1} / Q^{2}=4$ в зависимости от параметра $k$. В рассматриваемом течении псевдопластичные свойства жидкости в верхнем слое не изменяются, а изменяются в нижнем слое, определяемые параметром $k$. На рис. 4 приведены профили скорости для течения в случае $Q^{1} / Q^{2}=4, \mu_{0}^{1} / \mu_{0}^{2}=1, k^{1}=0.9, k^{2}=0.1,0.3,0.7,0.9$. Усиление псевдопластичных свойств (уменьшение показателя $k^{1}$ ) для нижнего слоя, приводит к уменьшению эффективной вязкости в этой области течения $\Omega^{1}$, особенно в областях больших значений градиента скорости. При течении жидкостей с разными расходами, но с одинаковыми псевдопластичными свойствами, в сечении на достаточно удаленном от точки схода потоков образуется параболический профиль скорости, характерный для однородного потока (рис. 4, кривая 4). Усиление же пседопластичных свойств, приводит к 
тому, что профиль скорости становится немонотонным, с образованием зоны изгиба на границе раздела слоев. Динамика развития установившегося профиля скорости для случая $Q^{1} / Q^{2}=4, \mu_{0}^{1} / \mu_{0}^{2}=1$, $k^{2}=0.9, k^{1}=0.3$ показана на рис. 5, где приведены профили скорости в сечениях $x_{1} / h=0.0$, $0.1,0.5,3.0$. В начальном сечении $x_{1} / h=0.0$ сходится два не ньютоновских потока с монотонными профилями скоростей.

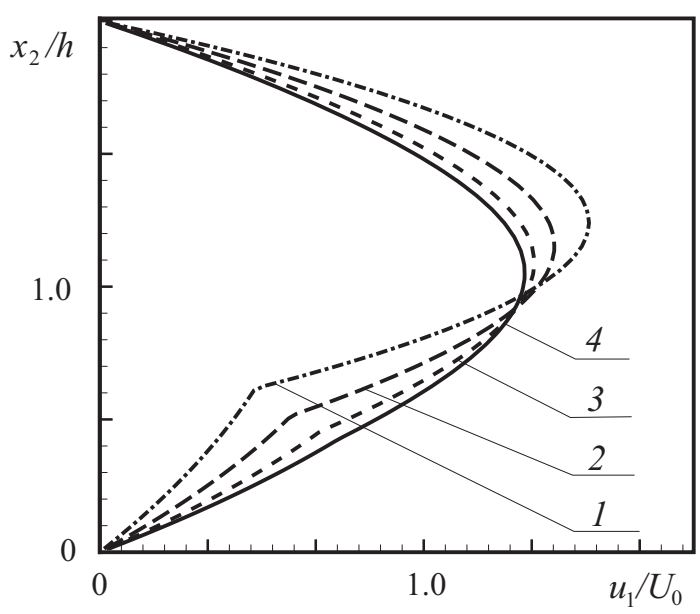

Рис. 4. Распределение скорости $u_{1} / U_{0}$ в сечении канала $x=3.0$ для $Q^{1} / Q^{2}=4, \mu_{0}^{1} / \mu_{0}^{2}=1, k^{1}=$ $=0.9$ при различных значениях $k^{2}: 1-k^{2}=0.1$, $2-k^{2}=0.3,3-k^{2}=0.7,4-k^{2}=0.9$

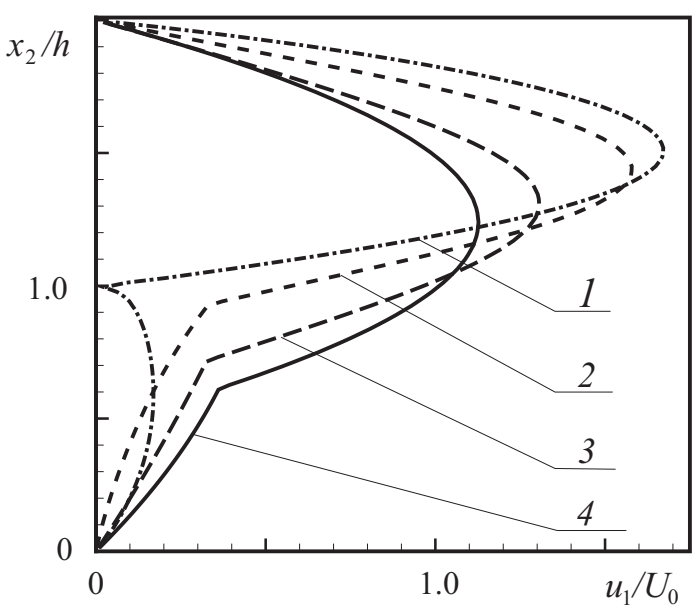

Рис. 5. Распределение скорости $u_{1} / U_{0}$ в вертикальных сечениях канала для $Q^{1} / Q^{2}=4$, $\mu_{0}^{1} / \mu_{0}^{2}=1, k^{1}=0.1, k^{2}=0.9: 1-x=1.0$; $2-x=1.1 ; 3-x=1.5 ; 4-x=3.0$

Из графиков видно, что в области схождения потоков начинается смещение границы раздела в область течения нижнего слоя, где течет жидкость с более псевдопластичными свойствами, характеризующимися меньшими значениями эффективной вязкости. Смещение границы в сторону нижней стенки приводит к расширению ширины потока верхнего слоя, что, в свою очередь, приводит к тому, что максимальные значения скорости уменьшаются, по мере удаления от точки схода потоков (рис. 5 , кривые $-2,3,4)$. Уменьшение максимального значения скорости сопровождается смещением данной зоны к нижней стенке, а в нижнем слое уменьшение ширины потока приводит к увеличению средней скорости потока. Отметим также, что двухслойное течение не ньютоновских жидкостей с большим отличием псевдопластичных свойств характеризуется тем, что расстояние, на котором происходит установление стационарного профиля скорости, увеличивается с ростом разницы псевдопластичных свойств.

На рис. 6 показана разность нормальных напряжений в канале фильеры $\tau_{11}-\tau_{22}$ в области течения для значений $Q^{1} / Q^{2}=4, \mu_{0}^{1} / \mu_{0}^{2}=1, k^{2}=0.9, k^{1}=0.3$. Видно, что на месте схождения потоков развиваются сильные нормальные напряжения, которые по мере продвижения вниз по потоку начинают релаксировать. Кроме того, наблюдаются отрицательные сжимающие напряжения вблизи поверхности раздела в области схождения потоков.

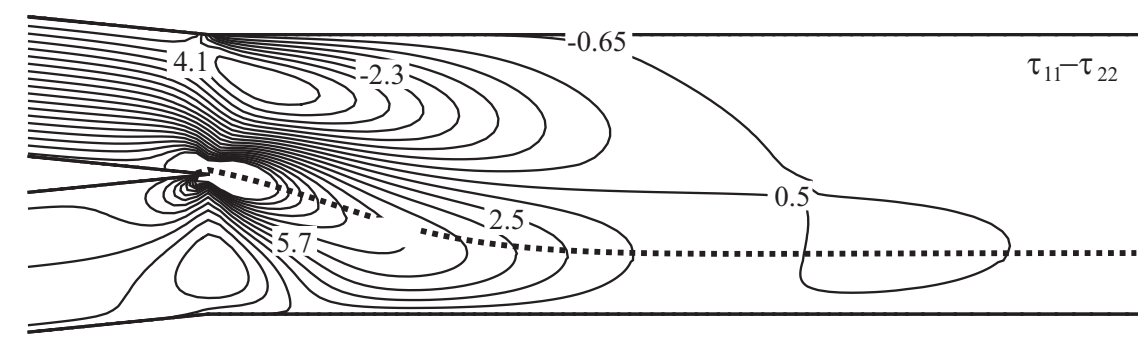

Рис. 6. Изолинии разницы напряжений $\tau_{11}-\tau_{22}$ в области схождения потоков для $Q^{1} / Q^{2}=4, \mu_{0}^{1} / \mu_{0}^{2}=1, k^{2}=0.9, k^{1}=0.3$ 


\title{
ЗАКЛЮЧЕНИЕ
}

В настоящей работе теоретически исследована структура течения и напряженно-деформированное состояние сред при двухслойном течении высоковязких не ньютоновских жидкостей в канале. Показано существенное влияние на положение границы раздела разницы величин отношения расхода жидкостей, показателей консистенции вязкости и степени псевдопластичности не ньютоновских сред. Исследовано влияние степени различия расходов, вязкостей, не ньютоновских свойств пседвопластичночти на структуру течения, гидродинамические характеристики двухслойного потока.

\section{Библиографический список}

1. Yih C. S. Instability due to viscosity stratification //

J. Fluid Mech. 1967. Vol. 27. P. 337-352.

2. Янков В. И., Глот И. О., Труфанова Н. М., Шакиров Н. В. Течение полимеров в отверстиях фильер. Теория, расчет, практика. М. ; Ижевск : Регулярная и хаотическая динамика, 2010. 368 с.

3. Хан Ч. Д. Реология в процессах переработки полимеров. М. : Химия, 1979. 366 с.

4. Володин В. П. Экструзия профильных изделий из термопластов. СПб. : Профессия, 2005. 480 с.

5. Bird R. B., Armstrong R. C., Hasager O. Dynamics of

Polymeric liquids. Vol 1. Fluid Mechanics. N. Y. : John Wiley ans Sons, 1987. 443 p.

6. Shikhmurzaev Y. D. Capillary Flows with Forming Interface. N. Y. : Chapman and Hall, 2008. 443 p.

7. Коннор Дж., Бреббиа К. Метод конечных элементов в механике жидкости. Л. : Судостроение, 1979. 264 с.

8. Снигерев Б. А., Тазюков Ф. Х. Об особенностях неизотермического обтекания сферы потоком вязкоупругой жидкости в стесненных условиях // Изв. Сарат. ун-та. Нов. сер. Сер. Математика. Механика. Информатика. 2011. Т. 11, вып. 1. С. 99-104.

\section{Double Layer of Polymer Melts in Channels of Dies}

\author{
B. A. Snigerev ${ }^{1}$, F. Kh. Tazyukov ${ }^{2}$ \\ ${ }^{1}$ Institute of Mechanics and Engineering, Kazan Science Center, Russian Academy of Sciences, 2/31, Lobachevsky str., Kazan, \\ 420111, Russia, Snigerev@mail.knc.ru \\ ${ }^{2}$ Kazan National Research Technological University, 68, K. Marxs str., Kazan, 420025, Russia, tazyukov@mail.ru \\ Numerical simulation of double-layer nonlinear viscous flow in channels of dies was performed. The fluid motion is described by \\ equations conservation of mass and momentum, supplemented by the rheological equation of state of nonlinear viscous fluid on the \\ Carreau model. The technique of numerical solve the problem based on the finite element method is described. Results the field of \\ velocities, pressure, stresses, position the interface boundary of two-layer flow depending on rheological properties of liquid and flow \\ regimes are presented.
}

Key words: non-newtonian fluid, interface boundary, twolayer flow.

\section{References}

1. Yih C. S. Instability due to viscosity stratification. J. Fluid Mech., 1967, vol. 27, pp. 337-352.

materials]. St. Petersburg, Profession, 2005, 480 p. (in

2. Jankov V. I., Glot I. O., Trufanova N. M., Shakirov N. V. Techenie polimerov v otverstijah fil'er. Teorija, raschet, praktika [For polymers in the holes of nozzles. Theory, computation practice]. Moscow, Izhevsk, Reguljarnaja i haoticheskaja dinamika, 2010, 368 p. (in Russian).

3. Han Ch. D. Reologija v processah pererabotki polimerov [Rheology in the plastics processing industry]. Moscow, Chemistry, 1979, 366 p. (in Russian).

4. Volodin V. P. Jekstruzija profil'nyh izdelij iz termoplastov [Extrusion profile products from thermoplastic Russian).

5. Bird R. B., Armstrong R. C., Hasager O. Dynamics of Polymeric liquids. Vol. 1. Fluid Mechanics. New York, John Wiley ans Sons, 1987, 443 p.

6. Shikhmurzaev Y. D. Capillary Flows with Forming Interface. New York, Chapman and Hall, 2008, 443 p.

7. Connor J., Brebbia C. Finite Element Techniques for Fluid Flow. London, Newnes-Butterworths, 1976.

8. Snigerev B. A., Tazyukov F. Kh. The feature of nonisothermal viscoelastic flows around sphere at obstruction condition. Izv. Saratov Univ. (N. S.), Ser. Math. Mech. Inform., 2011, vol. 11, iss. 1, pp. 99-104 (in Russian). 